Einschränkungen des Arztgeheimnisses werden längst nicht mehr ausschliesslich für die Behandlung potentiell gefährlicher Straftäter diskutiert: Auch Personen, deren Beruf Gelegenheit zum sogenannten erweiterten Suizid bietet oder Gewaltopfer sollen sich nicht mehr ihrem Arzt anvertrauen können, ohne die Weitergabe ihrer persönlichsten Informationen fürchten zu müssen. Wo ein Melderecht sinnvoll und wichtig ist, unterlaufen Meldepflichten berufsethische Prinzipien. Die nachstehende Stellungnahme der Ethikkommission der SAMW und der FMH zeigt diese Problematik auf und erläutert, warum Einschränkungen des Berufsgeheimnisses weder notwendig noch sinnvoll, sondern sogar kontraproduktiv sind und die öffentliche Sicherheit in keiner Weise erhöhen.

\title{
Berufsgeheimnis und Meldepflich- ten: Medizin-ethische Aspekte
}

FMH, SAMW

Verschiedene Entwicklungen tragen dazu bei, dass das medizinische Berufsgeheimnis zunehmend unter Druck gerät. So hat der tragische Tod der Sozialtherapeutin Adeline M. im Kanton Genf dazu geführt, dass die Kantone Genf, Wallis und Waadt [1] Gesetzesbestimmungen diskutieren, welche Ärzte (und weitere Medizinalpersonen) verpflichten, den Behörden alle Informationen über eine potentielle Gefährlichkeit ihrer Patienten im Strafvollzug weiterzugeben. Immer wieder schrecken auch Fälle von häuslicher Gewalt die Öffentlichkeit auf und die Politik sucht nach Lösungen. Im Kanton Basel-Landschaft [2] wird eine Gesetzesvorlage diskutiert, die Ärzte und weitere Personen, die einen Medizinal-, Psychologie- oder Gesundheitsberuf ausüben, dazu verpflichtet, bestimmte ärztliche Befunde, u.a. schwere Körperverletzungen, der Strafverfolgungsbehörde zu melden, unabhängig davon, ob diese durch Unfall, Delikt oder in suizidaler Absicht erfolgt sind. Zuletzt führte auch der tragische Absturz des Germanwings-Airbusses dazu, dass über Meldepflichten für dem Berufsgeheimnis unterstehende Personen diskutiert wird.

Das Anliegen, mit diesen Gesetzesänderungen die Interessen der Gesellschaft und die Sicherheit der Bürgerinnen und Bürger zu schützen und Risiken so weit wie möglich einzudämmen, ist nachvollziehbar. Zu prüfen ist aber, ob ärztliche Meldepflichten tatsächlich geeignet sind, die öffentliche Sicherheit zu erhöhen. Ausserdem sind mögliche langfristige Auswirkungen in die Abwägung einzubeziehen. $\mathrm{Zu}$ betonen ist, dass es eine «Null-Risiko-Gesellschaft» nicht gibt, auch wenn solche Ansprüche geäussert werden. Festzuhalten ist zudem, dass beim Tod von Adeline M. nicht das Arztgeheimnis, sondern Probleme im Vollzug eine Rolle gespielt haben. Der Untersuchungsbericht zum Fall [3] bedauert vielmehr den fehlenden Einbezug eines Arztes für die Risikoevaluation des Mörders von Adeline M. bei dessen Aufnahme in die Vollzugsanstalt.

$\mathrm{Zu}$ den Meldepflichten im Gefängnisbereich hat die Nationale Ethikkommission NEK eine ausführliche Stellungnahme [4] verfasst. Sie empfiehlt einstimmig, das heutige System - das bereits eine Meldemöglichkeit, aber keine Meldepflicht vorsieht - beizubehalten Als Gründe führt die Stellungnahme unter anderem an, dass

- eine Meldepflicht die Beurteilung der Gefährlichkeit nicht erleichtert und somit kein Instrument darstellt, um die Sicherheit der Bevölkerung zu verbessern;

- diese Meldepflicht im Gegenteil die Sicherheit gefährden kann, da inhaftierte Personen, die ihre Strafe verbüsst haben, in die Gesellschaft zurückkehren, ohne eine - vor allem im psychischen Bereich - angemessene Behandlung erhalten zu haben.

Die Zentrale Ethikkommission (ZEK) der Schweizerischen Akademie der Medizinischen Wissenschaften (SAMW) und die Verbindung der Schweizer Ärztinnen und Ärzte (FMH) schliessen sich den Argumenten der NEK an und erinnern nachstehend an die aus ihrer Sicht zentralen Werte:

\section{Medizinisches Berufsgeheimnis als berufsethischer Grundwert}

Das medizinische Berufsgeheimnis ist ein hohes Gut; dies wird dem Gesundheitsfachpersonal in der Aus-, Fort- und Weiterbildung als zentraler, berufsethischer 
Grundwert vermittelt. Das Berufsgeheimnis ist auch strafrechtlich geschützt, d.h., dessen Verletzung ist unter Strafe gestellt (Art. 321 StGB [5]). In Ausnahmesituationen ist die Offenbarung von Informationen durch Gesundheitsfachpersonen heute schon erlaubt. So besteht keine Strafbarkeit, wenn wichtige Informationen mit einer auf Gesuch erteilten, schriftlichen Bewilligung der vorgesetzten Behörde oder Aufsichtsbehörde oder in einer Notstandssituation weitergegeben werden [6].

\section{Medizinisches Berufsgeheimnis als notwendige Grundvoraussetzung einer therapeutischen Beziehung}

Die medizinische Behandlung erstreckt sich oft über längere Zeiträume. Insbesondere bei psychiatrischen und psychotherapeutischen Behandlungen, die eine Veränderung von Denken, Fühlen und Handeln anstreben, sind langfristige Erfolge nur in einem vertrauensvollen, geschützten Rahmen möglich. Patienten müssen die Möglichkeit haben, ihrem Therapeuten Dinge anzuvertrauen, die sie mit anderen Personen nicht besprechen können. Gleichzeitig sind diese Informationen auch Grundlage für die (psycho)therapeutische Arbeit. Werden solche Informationen zum eventuellen Schutz Dritter, aber auch zur Minimierung eigener Rechtsrisiken weitergegeben, besteht die Gefahr, dass sich Patienten nicht mehr öffnen und dem Therapeuten weniger oder verfälschte Einblicke in ihr Innenleben geben. Tritt dies ein, ist weder eine sinnvolle Therapie möglich, noch können in Notfallsituationen wichtige Hinweise weitergegeben werden, weil diese Informationen dem behandelnden Therapeuten fehlen.

\section{Medizinisches Berufsgeheimnis als Schutzraum für Opfer von Verbrechen oder Vergehen}

Opfer von Verbrechen oder Vergehen gegen Leib und Leben oder gegen die sexuelle Integrität benötigen einen therapeutischen Schutzraum und die Gewissheit, dass sie nicht aufgrund der Weitergabe von Informationen über ihren Kopf hinweg nochmals zum «Opfer» werden. Vielmehr müssen sie gemeinsam mit dem Arzt abwägen können, ob eine Meldung in ihrem Interesse ist und zu welchem Zeitpunkt sie erfolgen soll. Meldungen können kontraproduktiv sein und zu einer Retraumatisierung des Opfers führen, wenn sie z.B. ein Strafverfahren auslösen, in welchem der Täter aus Mangel an Beweisen freigesprochen wird. Analoge
Überlegungen gelten im Zusammenhang mit Kindern und Jugendlichen [7]. Eine Meldepflicht entspricht nicht immer dem Kindeswohl. Deshalb setzt sich auch die Stiftung Kinderschutz Schweiz für ein Melderecht des Arztes ein und spricht sich gegen eine Meldepflicht aus [8].

Zusammenfassend halten wir fest, dass die zunehmende Aufweichung des Berufsgeheimnisses weder notwendig noch sinnvoll ist und im Widerspruch zu den berufsethischen Prinzipien steht. Die bisher geltenden gesetzlichen Regelungen erlauben es den medizinisch und therapeutisch tätigen Berufspersonen heute schon, in Ausnahmesituationen den notwendigen Beitrag zur Sicherheit der Bürgerinnen und Bürger zu leisten, ohne ihren therapeutischen Auftrag zu gefährden. Die angestrebten Meldepflichten entsprechen keineswegs einem rationalen Umgang mit unvermeidlichen Risiken und tragen nichts zum Schutz der Gesellschaft bei. Eine Meldepflicht kann im Gegenteil kontraproduktiv wirken und verhindern, dass eine Gefahr für Drittpersonen rechtzeitig erkannt wird, weil sich diese Patienten gegenüber ihrer Ärztin oder ihrem Arzt aus Angst vor der Meldepflicht nicht mehr öffnen. Zudem verhindert sie dadurch eine optimale Therapie.

\section{Referenzen}

1 Projet de loi genevoise PL 11404 modifiant la loi d'application du code pénal et d'autres lois fédérales en matière pénale ( $\mathrm{LaCP}$ ) (E 4 10) du 27 août 2009; Projet de loi modifiant la loi du 4 juillet 2006 sur l'éxecution des condamnations pénales (LEP), Projet de loi valaisanne modifiant la loi d'application du code pénal suisse du 11 février 2009 (LACPP).

2 Änderung des Gesundheitsgesetzes (GesG): Schweigepflicht und Meldepflicht. Bericht an den Landrat des Kantons Basel-Landschaft vom 23. Februar 2015.

3 Ziegler Bernard, Avocat, Ancien Président du Conseil d'Etat: Rapport final dans l'enquête administrative ordonnée par le Conseil d'Etat à la suite du décès de Mme Adeline X lors d'une sortie accompagnée de M. Fabrice Anthamatten; 31 janvier 2014.

4 Nationale Ethikkommission im Bereich der Humanmedizin (2014) Über die Meldepflicht im Gefängnisbereich für Informationen, die dem medizinischen Berufsgeheimnis unterstehen. Stellung nahme Nr. 23/2014. www.nek-cne.ch/de/themen/stellungnahmen/ index.html

5 Art. 321 StGB, Verletzung des Berufsgeheimnisses: Ärzte, Zahnärzte, Apotheker, Hebammen sowie ihre Hilfspersonen, die ein Geheimnis offenbaren, das ihnen infolge ihres Berufes anvertraut worden ist oder das sie in dessen Ausübung wahrgenommen haben, werden, auf Antrag, mit Freiheitsstrafe bis zu drei Jahren oder Geldstrafe bestraft.

6 Art. 17 StGB, Rechtfertigender Notstand: Wer eine mit Strafe bedrohte Tat begeht, um ein eigenes oder das Rechtsgut einer anderen Person aus einer unmittelbaren, nicht anders abwendbaren Gefahr zu retten, handelt rechtmässig, wenn er dadurch höherwertige Interessen wahrt.

$7 \mathrm{Vgl}$. Stellungnahme gegen eine Meldepflicht und für ein Melderecht an die Erwachsenenschutzbehörden (KESB) der Stiftung Kindesschutz Schweiz im Rahmen der Vernehmlassung zur Änderung des Schweizerischen Zivilgesetzbuches, http://kinderschutz.ch/ $\mathrm{cmsn} / \mathrm{de}$

8 Stiftung Kindesschutz Schweiz, Vernehmlassungsantwort vom 10 März 2014 zur Änderung des Schweizerischen Zivilgesetzbuches. http://kinderschutz.ch/cmsn/de 